\title{
Sum-Rate Enhancement in Multiuser MIMO Decode-and-Forward Relay Broadcasting Channel with Energy Harvesting Relays
}

\author{
Fatma Benkhelifa, Ahmed Sultan Salem, and Mohamed-Slim Alouini \\ Computer, Electrical and Mathematical Science and Engineering (CEMSE) Division \\ King Abdullah University of Science and Technology (KAUST) \\ Thuwal, Makkah Province, Saudi Arabia \\ \{fatma.benkhelifa,ahmed.salem,slim.alouini\}@kaust.edu.sa
}

\begin{abstract}
In this paper, we consider a multiuser multipleinput multiple-output (MIMO) decode-and-forward (DF) relay broadcasting channel (BC) with single source, multiple energy harvesting (EH) relays and multiple destinations. All the nodes are equipped with multiple antennas. The EH and information decoding (ID) tasks at the relays and destinations are separated over the time, which is termed as the time switching (TS) scheme. As optimal solutions for the sum-rate maximization problems of BC channels and the MIMO interference channels are hard to obtain, the end-to-end sum rate maximization problem of a multiuser MIMO DF relay BC channel is even harder. In this paper, we propose to tackle a simplified problem where we employ the block diagonalization (BD) procedure at the source, and we mitigate the interference between the relaydestination channels using an algorithm similar to the BD method. In order to show the relevance of our low complex proposed solution, we compare it to the minimum mean-square error (MMSE) solution that was shown in the literature to be equivalent to the solution of the sum-rate maximization in MIMO broadcasting interfering channels. We also investigate the time division multiple access (TDMA) solution which separates all the information transmissions from the source to the relays and from the relays to the destinations over time. We provide numerical results to show the relevance of our proposed solution, in comparison with the no co-channel interference (CCI) case, the TDMA based solution and the MMSE based solution.
\end{abstract}

Index Terms-Energy harvesting, SWIPT, multiuser multipleinput multiple-output (MIMO), decode-and-forward (DF), broadcasting channel (BC), interference channel, sum-rate maximization, block diagonalization (BD), TDMA, MMSE.

\section{INTRODUCTION}

Simultaneous wireless information and power transfer (SWIPT) in wireless communication systems has recently gained significant research interest to study the simultaneous use of radio frequency (RF) signals to transmit data and to harvest energy. References [1] and [2] pioneered the study of SWIPT in single-input single-output (SISO) systems with flat-fading and frequency selective channels, respectively. The authors in [3] considered SWIPT in multiple-input multipleoutput (MIMO) systems and studied practical schemes that separate the energy harvesting $(\mathrm{EH})$ and the information decoding (ID) operations over the power domain or the time

This paper was funded by the Sensor Research Initiative funded by the Office of Sponsored Research at King Abdullah University of Science and Technology (KAUST), Thuwal, Makkah Province, Saudi Arabia. domain. These were called the power splitting (PS) and the time switching (TS) schemes, respectively.

Cooperative systems where SWIPT is employed were also studied since relay communications systems further improve the system capacity and communication coverage. In [4], the outage probability and the ergodic capacity of a twohop SISO amplify-and-forward (AF) relay system with an energy harvesting relay were investigated for delay-limited and delay-tolerant transmission modes. The authors in [5] extended this work to derive the achievable throughput of a continuous and discrete adaptive TS protocol where both AF and decode-and-forward (DF) relay networks were considered. In [6], a two-hop multi-antenna AF relay system with an independent multi-antenna EH receiver was considered where orthogonal space-time block codes (STBC) were employed for data transmission. The authors investigated the rate-energy tradeoff between the harvested energy at the EH receiver and the information rate at the destination node with knowledge of instantaneous channel state information (CSI), and the outage-energy tradeoff between the harvested energy at the EH receiver and the outage probability with second order statistics of the CSI. In [7], the achievable throughput and ergodic capacity were derived in a two-hop SISO DF relay system where the PS and TS schemes are employed.

On the other hand, multiuser diversity has been shown to further improve the performance of MIMO communication systems. The capacity of broadcasting channels has been studied in [8], [9]. In [10], dirty paper coding (DPC) was shown to be capacity achieving in MIMO broadcasting channels. However, DPC has a high computational complexity which made it hard to be implemented in practice. Several suboptimal algorithms with manageable complexity were proposed in the literature, such as the zero-forcing (ZF) and block diagonalization (BD) methods. When the receivers are equipped with multiple antennas, the $\mathrm{BD}$ method was proposed to eliminate the multi-user interference (MUI) by generalizing the ZF channel inversion method to the multiple antennas case [11]. The BD method was shown to have relatively low complexity while providing good performance. On the other hand, the multiuser MIMO interference channel (where the broadcasting channel is a special case) has also captured a lot of research interest. The sum rate maximization (MSR) problem of these channels was shown to be connected to 
the minimization of mean-square error (MSE) problem to obtain local optimums of the MSR problem [12]-[15]. In [13], a weighted sum rate maximization (MWSR) problem was considered and linear transceiver design algorithm was proposed based on the connection with iterative minimization of the weighted MSE. The proposed algorithm was shown to converge to a stationary point of the MWSR problem and was extended to a general class of sum-utility maximization problem.

Multiuser diversity where SWIPT is employed was studied in [16] where a one multi-antenna access point (AP) broadcasts wireless power to a set of single-antenna users via energy beamforming in the downlink (DL). The users then use the harvested energy to send back their information simultaneously to the AP in the uplink (UL). In this context, the DLUL time allocation, the DL energy beamforming and the UL power allocation are optimized to maximize the minimum uplink throughput among all users. The work done in [16] is extended in [17] to consider the massive MIMO (MM) case where the AP has a large number of antennas. The minimum transmission rate among all the users is maximized where the transmission frame is divided into the UL channel estimation phase, the DL energy transfer phase and the UL information transfer phase. In [18], the rate-energy region is investigated for a K-user MIMO interference channel where joint wireless information and energy transfer is considered in which the receivers either decodes the information or harvests the energy.

This paper fits into this line of research where the SWIPT technique is employed in a cooperative system with multiuser MIMO DF relay broadcasting channels where the relays have EH capabilities. This scenario may occur when the base station (BS) is communicating with end-users and the direct link suffers from severe path attenuation or shadowing. However, there are $\mathrm{EH}$ powerless relays close to the BS that are ready to assist the BS transmission to the end users using energies gained from the BS itself. To the best of our knowledge, the multiuser MIMO relay broadcasting channels with multiple EH relays and single/multiple destinations were not tackled before in the literature. As cited above, the maximization problem of the capacity of MIMO broadcasting channels has a high computational complexity. This makes the sum rate maximization problem of multiuser MIMO relay broadcasting channels hard to be solved. In this paper, we seek to enhance the sum of the source-destinations rates by using the BD method at the source, and managing the interference between the relays and the destinations in different ways. Our results show the relevance of our sum rate enhancement solution by comparing its performance to special cases and other standard solutions where: (a) no co-channel interference (CCI) occurs at the relays and the destinations, (b) the $\mathrm{BD}$ procedure is performed at the source and no CCI occurs at the destinations, (c) the time division multiple access (TDMA) method is employed in a way to separate over time the source to relays information transmissions and the relays to destinations information transmissions, (d) the iterative minimum mean square error (MMSE) precoder and decoder solutions which is known to be equivalent to the sum rate maximization problem of MIMO interfering broadcasting channel.

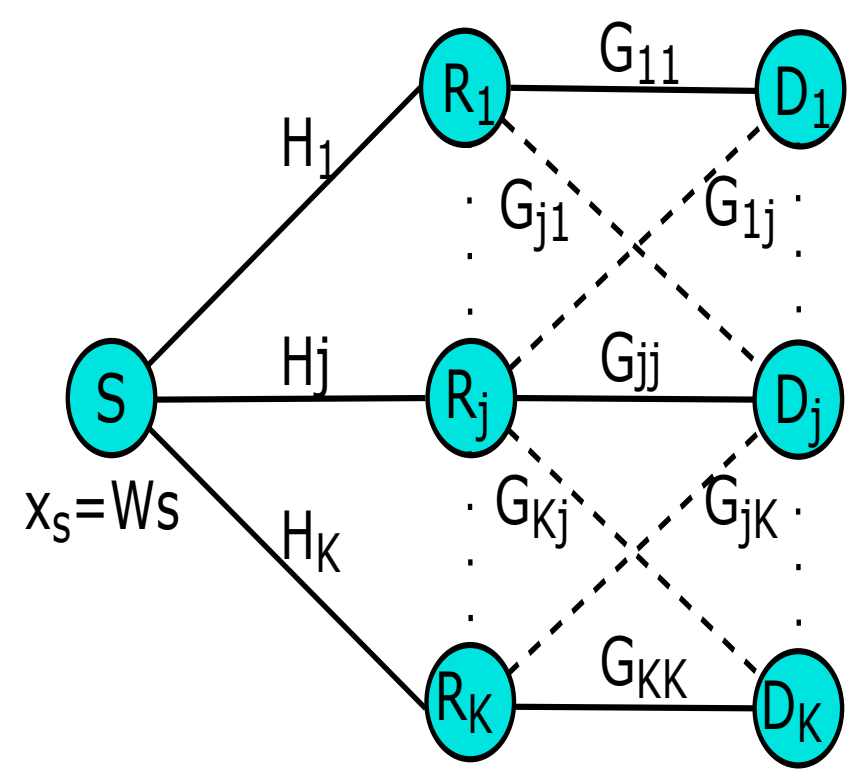

Figure 1. System model with one source, $K$ relays and $K$ destinations-all equipped with multiple antennas.

\section{System Model}

As shown in Fig. 1, we consider a two-hop MIMO DF relay communication system with one source $S$, multiple relays $R_{k, k=1, \ldots, K}$ and multiple destinations $D_{j, j=1, \ldots, K}$, with $K$ is the number of relays and destinations, $1 \leq k \leq K$ is the index of the relay, and $1 \leq j \leq K$ is the index of the destination. The source is equipped with $N_{s}$ antennas. Each relay $R_{k}$ and destination $D_{j}$ are equipped with $N_{r, k}$ and $N_{d, j}$ antennas, respectively. Let $N_{r}=\sum_{k=1}^{K} N_{r, k}$ and $N_{d}=\sum_{j=1}^{K} N_{d, j}$. We assume that $N_{s} \geq N_{r}$. The direct links between the source and the $K$ destinations are not considered, due to severe path attenuation and shadowing. Each relay operates in a halfduplex mode, i.e. the signals transmitted from the source to the $K$ relays and the signals transmitted from the $K$ relays to the $K$ destinations are transmitted orthogonally over time. The channel between the source and each relay $R_{k}$ and the channel between each relay $R_{k}$ and each destination $D_{j}$ are denoted as $\boldsymbol{H}_{k} \in \mathbb{C}^{N_{r, k} \times N_{s}}$ and $\boldsymbol{G}_{j, k} \in \mathbb{C}^{N_{d, j} \times N_{r, k}}$, respectively. All the channels $\boldsymbol{H}_{k}$ and $\boldsymbol{G}_{j, k}, \forall 1 \leq k \leq K$ and $1 \leq j \leq K$, are assumed to be quasi-static block-fading channels. We assume that the CSI is perfectly known at all the nodes.

While both the source and the destinations are battery powered, the relays are energy harvesting nodes. Each relay is equipped with an EH receiver and an ID receiver. We assume that the EH and ID receivers are operating at the same frequency. We also assume that the processing power used by the receive and transmit circuits at the relay is negligible compared to the transmit power of the relay.

The operations of the EH and ID receivers at the relays are separated via a time switcher that divides the transmission block into an energy harvesting phase and an information transmission phase. Let $\alpha$ be the time ratio allocated to the EH receiver at each relay, with $0 \leq \alpha \leq 1$.

- During the initial EH mode, the $K$ relays harvest energy 
from the received RF signal from the source during $\alpha T$ seconds.

- Then, the source sends the information carrying signal to the relays over a period of duration $\frac{1-\alpha}{2} T$ seconds.

- The relays use the harvested energy to forward the signal from the source to the destinations during the remaining part of the time slot.

Note that the TS scheme was chosen over the PS scheme only for its simplicity. The study of the PS scheme will be the object of future extension works. Without loss of generality, we assume $T=1$.

\section{A. Energy Harvesting at the Relays}

During the EH mode, the source transmits an energy carrying signal $\boldsymbol{x}_{e}$ of size $N_{s} \times 1$ to the $K$ relays, after precoding it, given by

$$
\boldsymbol{x}_{e}=\boldsymbol{W}_{e} \boldsymbol{s}_{e}=\left[\begin{array}{lll}
\boldsymbol{W}_{e, 1} & \ldots & \boldsymbol{W}_{e, K}
\end{array}\right]\left[\begin{array}{c}
\boldsymbol{s}_{e, 1} \\
\vdots \\
\boldsymbol{s}_{e, K}
\end{array}\right]=\sum_{k=1}^{K} \boldsymbol{W}_{e, k} \boldsymbol{s}_{e, k},
$$

where $\boldsymbol{s}_{e}$ is the $N_{r} \times 1$ signal transmitted to the $K$ relays with $\mathbb{E}\left[\boldsymbol{s}_{e} \boldsymbol{s}_{e}^{H}\right]=\boldsymbol{I}_{N_{r}}, \boldsymbol{W}_{e}$ is the associated precoding matrix at the source of size $N_{s} \times N_{r}, \boldsymbol{s}_{e, k}$ is the $N_{r, k} \times 1$ signal transmitted to the $k^{\prime}$ th relay, and $\boldsymbol{W}_{e, k}$ is the associated precoding matrix of the $k$ 'th relay. The covariance matrix of $\boldsymbol{x}_{e}$ is given by $\boldsymbol{S}_{e}=\mathbb{E}\left[\boldsymbol{x}_{e} \boldsymbol{x}_{e}^{H}\right]=\boldsymbol{W}_{e} \boldsymbol{W}_{e}^{H}$ and is subject to an average transmit constraint at the source. in other words, $\boldsymbol{S}_{e}$ satisfies: $\mathbb{E}\left[\left\|\boldsymbol{x}_{e}\right\|^{2}\right]=$ $\operatorname{tr}\left(\boldsymbol{S}_{e}\right) \leq P_{s}$ where $\operatorname{tr}($.$) denotes the trace operator.$

The $N_{r, k} \times 1$ received vector $\boldsymbol{y}_{e, r, k}$ at the $k$ 'th relay $R_{k}$, with $k=1, \ldots, K$, is given by

$$
\begin{aligned}
\boldsymbol{y}_{e, r, k}=\boldsymbol{H}_{k} \boldsymbol{x}_{e}+\boldsymbol{n}_{e, r, k} & =\boldsymbol{H}_{k}\left[\begin{array}{lll}
\boldsymbol{W}_{e, 1} & \ldots & \boldsymbol{W}_{e, K}
\end{array}\right]\left[\begin{array}{c}
\boldsymbol{s}_{e, 1} \\
\vdots \\
\boldsymbol{s}_{e, K}
\end{array}\right]+\boldsymbol{n}_{e, r, k} \\
& =\boldsymbol{H}_{k} \boldsymbol{W}_{e, k} \boldsymbol{s}_{e, k}+\boldsymbol{H}_{k} \sum_{l \neq k} \boldsymbol{W}_{e, l} \boldsymbol{s}_{e, l}+\boldsymbol{n}_{e, r, k},
\end{aligned}
$$

where $\boldsymbol{n}_{e, r, k}$ is the $N_{r, k} \times 1$ additive white Gaussian noise (AWGN) vector whose entries are independent identically distributed (i.i.d.) and drawn from the Gaussian distribution with zero mean and variance equal to $\sigma_{r, k}^{2}$. Once received, the EH receiver at each relay $R_{k}$ converts the RF received signal $\boldsymbol{y}_{e, r, k}$ to a direct current (DC) using a rectifier [19]. The harvested energy $Q_{r, k}$ at each relay $R_{k}$ (in unit of energy) can be written as

$$
Q_{r, k}\left(\alpha, \boldsymbol{S}_{e}\right)=\alpha \zeta \mathbb{E}\left[\left\|\boldsymbol{H}_{k} \boldsymbol{x}_{e}\right\|^{2}\right]=\alpha \zeta \operatorname{tr}\left(\boldsymbol{H}_{k} \boldsymbol{S}_{e} \boldsymbol{H}_{k}^{H}\right),
$$

where $\zeta \in[0,1]$ is the conversion efficiency.

\section{B. Source-to-Relays Data Transmission}

Once the energy is harvested at the $K$ relays, the source sends data streams to each destination via the relays after precoding them using matrix $\boldsymbol{W}_{s}$ of size $N_{s} \times n$, with $n=$
$\sum_{k=1}^{K} n_{k}=\sum_{k=1}^{K} \min \left(N_{r, k}, N_{d, k}\right)$. The $N_{s} \times 1$ transmitted signal at the source $\boldsymbol{x}_{s}$ is given by

$$
\boldsymbol{x}_{s}=\boldsymbol{W}_{s} \boldsymbol{s}=\left[\begin{array}{lll}
\boldsymbol{W}_{s, 1} & \ldots & \boldsymbol{W}_{s, K}
\end{array}\right]\left[\begin{array}{c}
\boldsymbol{s}_{1} \\
\vdots \\
\boldsymbol{s}_{K}
\end{array}\right]=\sum_{k=1}^{K} \boldsymbol{W}_{s, k} \boldsymbol{s}_{k},
$$

where $s$ is the $n \times 1$ data signal transmitted to the $K$ destinations

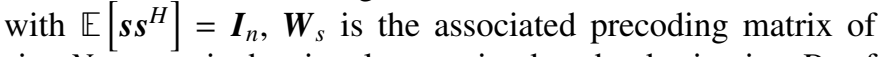
size $N_{s} \times n, \boldsymbol{s}_{k}$ is the signal transmitted to the destination $D_{k}$ of size $n_{k} \times 1$, and $\boldsymbol{W}_{s, k}$ is the associated precoding matrix of size $N_{s} \times n_{k}$. The covariance matrix of $\boldsymbol{x}_{s}$ is $\boldsymbol{S}=\mathbb{E}\left[\boldsymbol{x}_{s} \boldsymbol{x}_{s}^{H}\right]=\boldsymbol{W}_{s} \boldsymbol{W}_{s}^{H}$ and satisfies $\mathbb{E}\left[\left\|\boldsymbol{x}_{s}\right\|^{2}\right]=\operatorname{tr}(\boldsymbol{S}) \leq P_{s}$. The $N_{r, k} \times 1$ received vector $\boldsymbol{y}_{r, k}$ at the $k$ 'th relay $R_{k}$, with $k=1, \ldots, K$, is given by

$$
\begin{aligned}
\boldsymbol{y}_{r, k}=\boldsymbol{H}_{k} \boldsymbol{x}_{s}+\boldsymbol{n}_{r, k} & =\boldsymbol{H}_{k}\left[\begin{array}{lll}
\boldsymbol{W}_{s, 1} & \ldots & \boldsymbol{W}_{s, K}
\end{array}\right]\left[\begin{array}{c}
\boldsymbol{s}_{1} \\
\vdots \\
\boldsymbol{s}_{K}
\end{array}\right]+\boldsymbol{n}_{r, k} \\
& =\boldsymbol{H}_{k} \boldsymbol{W}_{s, k} \boldsymbol{s}_{k}+\boldsymbol{H}_{k} \sum_{l \neq k} \boldsymbol{W}_{s, l} \boldsymbol{s}_{l}+\boldsymbol{n}_{r, k},
\end{aligned}
$$

where $\boldsymbol{x}_{s}$ is the transmitted signal from the source defined in (5), $\boldsymbol{n}_{r, k}$ is the $N_{r, k} \times 1$ AWGN vector whose entries are i.i.d. and drawn from the Gaussian distribution with zero mean and variance equal to $\sigma_{r, k}^{2}$. We denote by $\boldsymbol{S}_{k}=\boldsymbol{W}_{s, k} \boldsymbol{W}_{s, k}^{H}$. The rate $R_{S-R_{k}}$ between the source and the $k^{\prime}$ th relay $R_{k}$ is given by [20]

$$
\begin{aligned}
R_{S-R_{k}}(\alpha, \boldsymbol{S}) & =\frac{1-\alpha}{2} \log _{2}\left|\boldsymbol{I}_{N_{r, k}}+\boldsymbol{R}_{-k}^{-1} \boldsymbol{H}_{k} \boldsymbol{S}_{k} \boldsymbol{H}_{k}^{H}\right| \\
& =\frac{1-\alpha}{2} \log _{2}\left|\boldsymbol{I}_{N_{r, k}}+\boldsymbol{R}_{-k}^{-1} \boldsymbol{H}_{k} \boldsymbol{W}_{s, k} \boldsymbol{W}_{s, k}^{H} \boldsymbol{H}_{k}^{H}\right|,
\end{aligned}
$$

where

$$
\boldsymbol{R}_{-k}=\sigma_{r, k}^{2} \boldsymbol{I}_{N_{r, k}}+\sum_{l \neq k} \boldsymbol{H}_{k} \boldsymbol{S}_{l} \boldsymbol{H}_{k}^{H}=\sigma_{r, k}^{2} \boldsymbol{I}_{N_{r, k}}+\sum_{l \neq k} \boldsymbol{H}_{k} \boldsymbol{W}_{s, l} \boldsymbol{W}_{s, l}^{H} \boldsymbol{H}_{k}^{H} .
$$

\section{Relays-to-Destinations Data Transmission}

During the next $\frac{1-\alpha}{2}$ seconds of the ID period, each relay $R_{k}$ forwards the decoded signal using $\boldsymbol{y}_{r, k}$ after precoding it. The signal emitted by each relay $R_{k}$ is $\boldsymbol{x}_{r, k}=\boldsymbol{W}_{r, k} \boldsymbol{s}_{k}$, which has a covariance matrix given by the $N_{r, k} \times N_{r, k}$ matrix $\boldsymbol{R}_{r, k}=$ $\boldsymbol{W}_{r, k} \boldsymbol{W}_{r, k}^{H}$. The relay is constrained to an average transmit power constraint given by

$$
\mathbb{E}\left[\boldsymbol{x}_{r, k} \boldsymbol{x}_{r, k}^{H}\right]=\operatorname{tr}\left(\boldsymbol{R}_{r, k}\right) \leq P_{r, k}\left(\alpha, \boldsymbol{S}_{e}\right),
$$

where $P_{r, k}\left(\alpha, \boldsymbol{S}_{e}\right)=\frac{2}{1-\alpha} Q_{r, k}\left(\alpha, \boldsymbol{S}_{e}\right)=\frac{2 \alpha \zeta}{1-\alpha} \operatorname{tr}\left(\boldsymbol{H}_{k} \boldsymbol{S}_{e} \boldsymbol{H}_{k}^{H}\right)$. The $N_{d, k} \times 1$ received vector $\boldsymbol{y}_{d, k}$ at the $k^{\prime}$ th destination $D_{k}$, with $k=1, \ldots, K$, is expressed as

$$
\begin{aligned}
\boldsymbol{y}_{d, k} & =\boldsymbol{G}_{k, k} \boldsymbol{x}_{r, k}+\sum_{l \neq k} \boldsymbol{G}_{k, l} \boldsymbol{x}_{r, l}+\boldsymbol{n}_{d, k} \\
& =\boldsymbol{G}_{k, k} \boldsymbol{W}_{r, k} \boldsymbol{s}_{k}+\sum_{l \neq k} \boldsymbol{G}_{k, l} \boldsymbol{W}_{r, l} \boldsymbol{s}_{l}+\boldsymbol{n}_{d, k},
\end{aligned}
$$

where $\boldsymbol{n}_{d, k}$ is the $N_{d, k} \times 1$ AWGN vector whose entries are i.i.d. and drawn from the Gaussian distribution with zero mean and 
variance equal to $\sigma_{d, k}^{2}$. Consequently, the rate $R_{S_{k}-R_{k}}$ between the $k^{\prime}$ th relay $R_{k}$ and the $k^{\prime}$ th destination $D_{k}$ is given by:

$$
\begin{aligned}
R_{R_{k}-D_{k}}\left(\alpha, \boldsymbol{R}_{r}\right) & =\frac{1-\alpha}{2} \log _{2}\left|\boldsymbol{I}_{N_{d, k}}+\boldsymbol{M}_{-k}^{-1} \boldsymbol{G}_{k, k} \boldsymbol{R}_{r, k} \boldsymbol{G}_{k, k}^{H}\right| \\
& =\frac{1-\alpha}{2} \log _{2}\left|\boldsymbol{I}_{N_{d, k}}+\boldsymbol{M}_{-k}^{-1} \boldsymbol{G}_{k, k} \boldsymbol{W}_{r, k} \boldsymbol{W}_{r, k}^{H} \boldsymbol{G}_{k, k}^{H}\right|,
\end{aligned}
$$

where

$$
\begin{aligned}
\boldsymbol{M}_{-k} & =\sigma_{d, k}^{2} \boldsymbol{I}_{N_{d, k}}+\sum_{l \neq k} \boldsymbol{G}_{k, l} \boldsymbol{R}_{r, l} \boldsymbol{G}_{k, l}^{H} \\
& =\sigma_{d, k}^{2} \boldsymbol{I}_{N_{d, k}}+\sum_{l \neq k} \boldsymbol{G}_{k, l} \boldsymbol{W}_{r, l} \boldsymbol{W}_{r, l}^{H} \boldsymbol{G}_{k, l}^{H} .
\end{aligned}
$$

Note that the $k^{\prime}$ th destination is only interested in decoding the data transmitted from the $k^{\prime}$ th relay. Let $\boldsymbol{R}_{r}=\left[\begin{array}{c}R_{r, 1} \\ \vdots \\ R_{r, K}\end{array}\right]$ be the vertical concatenation of all the matrices $\boldsymbol{R}_{r, k}, \forall 1 \leq k \leq K$.

\section{Precoders Design at the Source and Relays}

The objective of this work is to characterize the design of the optimal covariance matrices $\boldsymbol{S}_{e}$ and $\boldsymbol{S}$ at the source during the EH period and ID period, respectively, and the design of the optimal covariance matrices $\boldsymbol{R}_{r, k}$ at the $K$ relays in order to maximize the sum of the end-to-end transmission rates. The sum of the source-destinations transmission rates of the MIMO $\mathrm{DF}$ relay system, in bits $/ \mathrm{s} / \mathrm{Hz}$, is given by

$$
R\left(\alpha, \boldsymbol{S}, \boldsymbol{R}_{r}\right)=\sum_{k=1}^{K} \min \left(R_{S-R_{k}}(\alpha, S), R_{R_{k}-D_{k}}\left(\alpha, \boldsymbol{R}_{r}\right)\right) .
$$

The optimal design of $\alpha, \boldsymbol{S}_{e}, \boldsymbol{S}$ and $\boldsymbol{R}_{r, k}$ corresponds to the following maximization problem of the sum of the sourcedestinations transmission rates, subject to the transmit power constraint at the source $P_{s}$ and the transmit power constraint at the $K$ relays $P_{r, k}$ :

(P) $\max _{\alpha, \boldsymbol{S}_{e}, \boldsymbol{S}, \boldsymbol{R}_{r}} R\left(\alpha, \boldsymbol{S}, \boldsymbol{R}_{r}\right)=\sum_{k=1}^{K} \min \left(R_{S-R_{k}}(\alpha, \boldsymbol{S}), R_{R_{k}-D_{k}}\left(\alpha, \boldsymbol{R}_{r}\right)\right)$,

$$
\begin{array}{ll}
\text { s.t. } & \operatorname{tr}(\boldsymbol{S}) \leq P_{s}, \\
& \operatorname{tr}\left(\boldsymbol{S}_{e}\right) \leq P_{s}, \\
& \operatorname{tr}\left(\boldsymbol{R}_{r, k}\right) \leq P_{r, k}\left(\alpha, \boldsymbol{S}_{e}\right), \\
& \boldsymbol{S} \geq 0, \boldsymbol{S}_{e} \geq 0, \boldsymbol{R}_{r, k} \geq 0, \\
& 0 \leq \alpha \leq 1 .
\end{array}
$$

This optimization problem is not convex since the objective function (19a) is not concave, and hence the optimal solution is hard to obtain. In order to solve the problem (P), we fix $\alpha \in[0,1]$ and we solve the problem for a given $\alpha$. Recall that the maximum of a multivariable objective function can be computed as $\max _{x, y} f(x, y)=\max _{x} \max _{y} f(x, y)$ [21]. Then, the optimal $\alpha$ can be easily obtained using a one-dimensional search method, either the greedy search or the bisection method [22]. Note that the optimal $\alpha$ satisfies: $0<\alpha<1$. In our work, we propose to tackle the simplified sum-rate enhancement problem explained below:

- We fix the value of $0<\alpha<1$.
- We use BD at the source to eliminate the interference induced by one relay's signals on the others and we derive the structure of the covariance matrix $S$ at the source during the information transmission period.

- We derive the structure of the covariance matrices $\boldsymbol{R}_{r, k}$ at the $K$ relays via maximizing the sum rate of the interference channels between the relays and the destinations,

- Either, by using the recursive update of the matrices $\boldsymbol{M}_{-k}$, until convergence.

- Or, by canceling the interference between the destinations using a technique similar to the BD method.

- Using these three steps, Problem (P) simplifies to an equivalent optimization problem that is convex and can be jointly optimized.

- We jointly optimize the covariance matrix $\boldsymbol{S}_{e}$ at the source during the energy harvesting, with the obtained covariance matrices $\boldsymbol{S}$ and $\boldsymbol{R}_{r, k}$ from the previous steps, for a given $\alpha \in(0,1)$.

- We obtain the optimal $\alpha$ via a one-dimensional search method.

In what follows, we detail this outline and present the solutions.

\section{A. Block Diagonalization at the Source}

During the first $\frac{1-\alpha}{2} T$ of the ID period, the $N_{r, k} \times 1$ received vector $\boldsymbol{y}_{r, k}$ at each relay $R_{k}$ is given by:

$$
\boldsymbol{y}_{r, k}=\boldsymbol{H}_{k} \boldsymbol{W}_{s, k} \boldsymbol{s}_{k}+\boldsymbol{H}_{k} \sum_{l \neq k} \boldsymbol{W}_{s, l} \boldsymbol{s}_{l}+\boldsymbol{n}_{r, k} .
$$

In order to eliminate the interference induced by one relay on the others, we use BD where the precoders $\boldsymbol{W}_{s, k}$ are chosen to satisfy

$$
\boldsymbol{H}_{l} \boldsymbol{W}_{s, k}=0, \forall k \neq l .
$$

Let $\boldsymbol{H}_{-k}$ denote the $\left(N_{r}-N_{r, k}\right) \times N_{s}$ matrix such that $\boldsymbol{H}_{-k}$ is the vertical concatenation of all the $\boldsymbol{H}_{l}$ except $\boldsymbol{H}_{k}$. The BD conditions are equivalent to

$$
\boldsymbol{H}_{-k} \boldsymbol{W}_{s, k}=0, \forall k \text {. }
$$

Let us consider the singular value decomposition (SVD) of $\boldsymbol{H}_{-k}$.

$$
\boldsymbol{H}_{-k}=\boldsymbol{U}_{s,-k} \boldsymbol{D}_{s,-k}^{1 / 2}\left(\boldsymbol{V}_{s,-k}\right)^{H}=\boldsymbol{U}_{s,-k} \boldsymbol{D}_{s,-k}^{1 / 2}\left[\begin{array}{ll}
\boldsymbol{V}_{s,-k}^{a} & \boldsymbol{V}_{s,-k}^{b}
\end{array}\right]^{H},
$$

where $\boldsymbol{U}_{s,-k}, \boldsymbol{V}_{s,-k}, \boldsymbol{V}_{s,-k}^{a}$ and $\boldsymbol{V}_{s,-k}^{b}$ are unitary matrices with dimensions $\left(N_{r}-N_{r, k}\right) \times\left(N_{r}-N_{r, k}\right), N_{s} \times N_{s}, N_{s} \times \tilde{r}_{k}$ and $N_{s} \times$ $\left(N_{s}-\tilde{r}_{k}\right)$, respectively, $\boldsymbol{D}_{s,-k}$ is the diagonal matrix containing the eigenvalues of $\boldsymbol{H}_{-k} \boldsymbol{H}_{-k}^{H}$ arranged in a decreasing order, and $\tilde{r}_{k}$ is the rank of $\boldsymbol{H}_{-k} . \boldsymbol{V}_{s,-k}^{a}$ and $\boldsymbol{V}_{s,-k}^{b}$ contain the eigenvectors of $\boldsymbol{H}_{-k} \boldsymbol{H}_{-k}^{H}$ corresponding to its nonzero eigenvalues and zero eigenvalues, respectively. Note that in order to satisfy the BD conditions, we should have the dimension condition $\tilde{r}_{k}<N_{s}$ [11]. Now, let us consider the SVD of the $N_{r, k} \times\left(N_{s}-\tilde{r}_{k}\right)$ matrix $\boldsymbol{H}_{k} \boldsymbol{V}_{s,-k}^{b}$ :

$$
\begin{aligned}
\boldsymbol{H}_{k} \boldsymbol{V}_{s,-k}^{b} & =\tilde{\boldsymbol{U}}_{s,-k}\left[\begin{array}{cc}
\tilde{\boldsymbol{D}}_{s,-k}^{1 / 2} & 0 \\
0 & 0
\end{array}\right]\left(\tilde{\boldsymbol{V}}_{s,-k}\right)^{H} \\
& =\tilde{\boldsymbol{U}}_{s,-k}\left[\begin{array}{cc}
\tilde{\boldsymbol{D}}_{s,-k}^{1 / 2} & 0 \\
0 & 0
\end{array}\right]\left[\begin{array}{ll}
\tilde{\boldsymbol{V}}_{s,-k}^{a} & \tilde{\boldsymbol{V}}_{s,-k}^{b}
\end{array}\right]^{H},
\end{aligned}
$$


where $\tilde{\boldsymbol{U}}_{s,-k}, \tilde{\boldsymbol{V}}_{s,-k}, \tilde{\boldsymbol{V}}_{s,-k}^{a}$ and $\tilde{\boldsymbol{V}}_{s,-k}^{b}$ are unitary matrices with dimensions $N_{r, k} \times N_{r, k},\left(N_{s}-\tilde{r}_{k}\right) \times\left(N_{s}-\tilde{r}_{k}\right),\left(N_{s}-\tilde{r}_{k}\right) \times \bar{r}_{k}$ and $\left(N_{s}-\tilde{r}_{k}\right) \times\left(N_{s}-\tilde{r}_{k}-\bar{r}_{k}\right)$, respectively, $\tilde{\boldsymbol{D}}_{s,-k}$ is the $\bar{r}_{k} \times \bar{r}_{k}$ diagonal matrix containing the eigenvalues of $\boldsymbol{H}_{k} \boldsymbol{V}_{s,-k}^{b}$ arranged in a decreasing order, and $\bar{r}_{k}$ is the rank of $\boldsymbol{H}_{k} \boldsymbol{V}_{s,-k}^{b}\left(\boldsymbol{H}_{k} \boldsymbol{V}_{s,-k}^{b}\right)^{H}$. $\tilde{\boldsymbol{V}}_{s,-k}^{a}$ and $\tilde{\boldsymbol{V}}_{s,-k}^{b}$ contain the eigenvectors of $\boldsymbol{H}_{k} \boldsymbol{V}_{s,-k}^{b}\left(\boldsymbol{H}_{k} \boldsymbol{V}_{s,-k}^{b}\right)^{H}$ corresponding to its nonzero eigenvalues and zero eigenvalues, respectively.

Thus, the precoding matrices $\boldsymbol{W}_{s, k}$ are given by

$$
\boldsymbol{W}_{s, k}=\boldsymbol{V}_{s,-k}^{b} \tilde{\boldsymbol{V}}_{s,-k}^{a} \boldsymbol{D}_{W, s, k}^{1 / 2},
$$

where $\boldsymbol{D}_{W, s, k}^{1 / 2}$ is the $\bar{r}_{k} \times \bar{r}_{k}$ diagonal matrix. Given these expressions of $\boldsymbol{W}_{s, k}$, the corresponding rate between the source and the $k^{\prime}$ th relay $R_{k}$ can be rewritten as

$$
\begin{aligned}
\bar{R}_{S-R_{k}}\left(\alpha, \boldsymbol{D}_{W, s, k}\right) & =\frac{1-\alpha}{2} \log _{2}\left|\boldsymbol{I}+\frac{1}{\sigma_{r, k}^{2}} \boldsymbol{H}_{k} \boldsymbol{W}_{s, k} \boldsymbol{W}_{s, k}^{H} \boldsymbol{H}_{k}^{H}\right| \\
& =\frac{1-\alpha}{2} \log _{2}\left|\boldsymbol{I}+\frac{1}{\sigma_{r, k}^{2}} \tilde{\boldsymbol{D}}_{s,-k} \boldsymbol{D}_{W, s, k}\right| .
\end{aligned}
$$

\section{B. Multi-User Interference Mitigation at the Destinations}

After the use of BD at the source, the optimization problem that maximizes the sum of the achievable rates between the source and the destinations becomes:

$$
\begin{aligned}
(\mathrm{P} 1): \max _{\boldsymbol{S}_{e}, \boldsymbol{D}_{W, s, k}, \boldsymbol{R}_{r}} & \sum_{k=1}^{K} \min \left(\bar{R}_{S-R_{k}}\left(\alpha, \boldsymbol{D}_{W, s, k}\right), R_{R_{k}-D_{k}}\left(\alpha, \boldsymbol{R}_{r}\right)\right), \\
\text { s.t. } & \sum_{k=1}^{K} \operatorname{tr}\left(\boldsymbol{D}_{W, s, k}\right) \leq P_{s}, \\
& \operatorname{tr}\left(\boldsymbol{R}_{r, k}\right) \leq P_{r, k}\left(\alpha, \boldsymbol{S}_{e}\right) \\
& \operatorname{tr}\left(\boldsymbol{S}_{e}\right) \leq P_{s} \\
& \boldsymbol{D}_{W, s, k} \geq 0, \boldsymbol{R}_{r, k} \geq 0, \boldsymbol{S}_{e} \geq 0
\end{aligned}
$$

This problem is still not convex, since the objective function is not concave. The nonconvexity of the problem is due to the matrices $\boldsymbol{M}_{-k}$, which are related to the interference between the relay-destination channels.

We propose to solve $(\mathrm{P} 1)$ using a method similar to the BD method used in the first phase of data transmission. Recall, the received vector $\boldsymbol{y}_{d, k}$ at each destination $D_{k}$ is given by

$$
\begin{aligned}
\boldsymbol{y}_{d, k} & =\boldsymbol{G}_{k, k} \boldsymbol{x}_{r, k}+\sum_{l \neq k} \boldsymbol{G}_{k, l} \boldsymbol{x}_{r, l}+\boldsymbol{n}_{d, k} \\
& =\boldsymbol{G}_{k, k} \boldsymbol{W}_{r, k} \boldsymbol{s}_{k}+\sum_{l \neq k} \boldsymbol{G}_{k, l} \boldsymbol{W}_{r, l} \boldsymbol{s}_{l}+\boldsymbol{n}_{d, k} .
\end{aligned}
$$

Here in order to eliminate the interference between the destinations, we can choose the precoders $\boldsymbol{W}_{r, k}$ such that

$$
\boldsymbol{G}_{l, k} \boldsymbol{W}_{r, k}=0, \forall l \neq k \text {. }
$$

Let $\boldsymbol{G}_{-k, k}$ denotes the $\left(N_{d}-N_{d, k}\right) \times N_{r, k}$ matrix as the concatenation of all the matrices $\boldsymbol{G}_{l, k}$ except $\boldsymbol{G}_{k, k}$. The BD conditions are given by

$$
\boldsymbol{G}_{-k, k} \boldsymbol{W}_{r, k}=0 .
$$

Let us consider the following SVD decompositions

$$
\boldsymbol{G}_{-k, k}=\boldsymbol{U}_{r,-k} \boldsymbol{D}_{r,-k}^{1 / 2}\left(\boldsymbol{V}_{r,-k}\right)^{H}=\boldsymbol{U}_{r,-k} \boldsymbol{D}_{r,-k}^{1 / 2}\left[\begin{array}{ll}
\boldsymbol{V}_{r,-k}^{a} & \boldsymbol{V}_{r,-k}^{b}
\end{array}\right]^{H},
$$

$$
\begin{aligned}
\boldsymbol{G}_{k, k} \boldsymbol{V}_{r,-k}^{b} & =\tilde{\boldsymbol{U}}_{r,-k}\left[\begin{array}{cc}
\tilde{\boldsymbol{D}}_{r,-k}^{1 / 2} & 0 \\
0 & 0
\end{array}\right]\left(\tilde{\boldsymbol{V}}_{r,-k}\right)^{H} \\
& =\tilde{\boldsymbol{U}}_{r,-k}\left[\begin{array}{cc}
\tilde{\boldsymbol{D}}_{r,-k}^{1 / 2} & 0 \\
0 & 0
\end{array}\right]\left[\begin{array}{ll}
\tilde{\boldsymbol{V}}_{r,-k}^{a} & \tilde{\boldsymbol{V}}_{r,-k}^{b}
\end{array}\right]^{H},
\end{aligned}
$$

where $\boldsymbol{U}_{r,-k}, \boldsymbol{V}_{r,-k}, \boldsymbol{V}_{r,-k}^{a}, \boldsymbol{V}_{r,-k}^{b}, \tilde{\boldsymbol{U}}_{r,-k}, \tilde{\boldsymbol{V}}_{r,-k}, \tilde{\boldsymbol{V}}_{r,-k}^{a}$ and $\tilde{\boldsymbol{V}}_{r,-k}^{b}$ are unitary matrices with dimensions $\left(N_{d}-N_{d, k}\right) \times\left(N_{d}-N_{d, k}\right)$, $N_{r, k} \times N_{r, k}, N_{r, k} \times \tilde{g}_{k}, N_{r, k} \times\left(N_{r, k}-\tilde{g}_{k}\right), N_{d, k} \times N_{d, k},\left(N_{r, k}-\tilde{g}_{k}\right) \times$ $\left(N_{r, k}-\tilde{g}_{k}\right),\left(N_{r, k}-\tilde{g}_{k}\right) \times \bar{g}_{k}$ and $\left(N_{t, k}-\tilde{g}_{k}\right) \times\left(N_{r, k}-\tilde{g}_{k}-\bar{g}_{k}\right)$, respectively, $\boldsymbol{D}_{r,-k}$ and $\tilde{\boldsymbol{D}}_{r,-k}$ are the diagonal matrices containing the eigenvalues arranged in a decreasing order of $\boldsymbol{G}_{-k, k} \boldsymbol{G}_{-k, k}^{H}$ and $\boldsymbol{G}_{k, k} \boldsymbol{V}_{r,-k}^{b}$, respectively and $\tilde{g}_{k}$ and $\bar{g}_{k}$ are the ranks of $\boldsymbol{G}_{-k, k}$ and $\boldsymbol{G}_{k, k} \boldsymbol{V}_{r,-k}^{b}\left(\boldsymbol{G}_{k, k} \boldsymbol{V}_{r,-k}^{b}\right)^{H}$, respectively. $\boldsymbol{V}_{g,-k}^{a}$ and $\boldsymbol{V}_{g,-k}^{b}$ contain the eigenvectors of $\boldsymbol{G}_{-k, k} \boldsymbol{G}_{-k, k}^{H}$ corresponding to its nonzero eigenvalues and zero eigenvalues, respectively. $\tilde{\boldsymbol{V}}_{r,-k}^{a}$ and $\tilde{\boldsymbol{V}}_{r,-k}^{b}$ contain the eigenvectors of $\boldsymbol{G}_{k, k} \boldsymbol{V}_{r,-k}^{b}\left(\boldsymbol{G}_{k, k} \boldsymbol{V}_{r,-k}^{b}\right)^{H}$ corresponding to its nonzero eigenvalues and zero eigenvalues, respectively. Here, also, we should have the dimension condition $\tilde{g}_{k}<N_{r, k}$ in order to guarantee the BD conditions.

Similarly, as in section III-A, we show that the precoders $\boldsymbol{W}_{r, k}$ are given by

$$
\boldsymbol{W}_{r, k}=\boldsymbol{V}_{r,-k}^{b} \tilde{\boldsymbol{V}}_{r,-k}^{a} \boldsymbol{D}_{W, r, k}^{1 / 2},
$$

where $\boldsymbol{D}_{W, r, k}^{1 / 2}$ is the $\bar{g}_{k} \times \bar{g}_{k}$ diagonal matrix. Given these expressions of $\boldsymbol{W}_{r, k}$, the rate between the relay $R_{k}$ and the destination $D_{k}$ becomes given by

$$
\begin{aligned}
\bar{R}_{R_{k}-D_{k}}\left(\alpha, \boldsymbol{D}_{W, r, k}\right) & =\frac{1-\alpha}{2} \log _{2}\left|\boldsymbol{I}+\frac{1}{\sigma_{d, k}^{2}} \boldsymbol{G}_{k, k} \boldsymbol{W}_{r, k} \boldsymbol{W}_{r, k}^{H} \boldsymbol{G}_{k, k}^{H}\right| \\
& =\frac{1-\alpha}{2} \log _{2}\left|\boldsymbol{I}+\frac{1}{\sigma_{d, k}^{2}} \tilde{\boldsymbol{D}}_{r,-k} \boldsymbol{D}_{W, r, k}\right|,
\end{aligned}
$$

and hence the resolution of (P1) becomes simple and can be done by CVX software.

\section{Summary of Proposed Suboptimal Solution}

After employing BD for source-relays communications and relays-destinations communications, the optimization problem that maximizes the sum of the achievable rates between the source and the destinations is equivalent to

$$
\begin{aligned}
(\mathrm{P} 2): \max _{\boldsymbol{D}_{W, s,}, \boldsymbol{D}_{W, r, k}} & \sum_{k=1}^{K} \min \left(\bar{R}_{S-R_{k}}\left(\alpha, \boldsymbol{D}_{W, s, k}\right), \bar{R}_{R_{k}-D_{k}}\left(\alpha, \boldsymbol{D}_{W, r, k}\right)\right), \\
\text { s.t. } & \sum_{k=1}^{K} \operatorname{tr}\left(\boldsymbol{D}_{W, s, k}\right) \leq P_{s}, \\
& \operatorname{tr}\left(\boldsymbol{D}_{W, r, k}\right) \leq P_{r, k}\left(\alpha, \boldsymbol{S}_{e}\right), \\
& \operatorname{tr}\left(\boldsymbol{S}_{e}\right) \leq P_{s}, \\
& \boldsymbol{D}_{W, s, k} \geq 0, \boldsymbol{D}_{W, r, k} \geq 0, \boldsymbol{S}_{e} \geq 0 .
\end{aligned}
$$


In order to solve it, we proceed as follow. Let $t_{k}$ be a positive variable such that

$$
\begin{aligned}
& \text { (P3) : } \max _{\boldsymbol{S}_{e}, \boldsymbol{D}_{W, s, k}, \boldsymbol{D}_{W, r, k}} \sum_{k=1}^{K} \frac{1-\alpha}{2} t_{k}, \\
& \text { s.t. } t_{k} \leq \bar{R}_{S-R_{k}}\left(\alpha, \boldsymbol{D}_{W, s, k}\right), \\
& t_{k} \leq \bar{R}_{R_{k}-D_{k}}\left(\alpha, \boldsymbol{D}_{W, r, k}\right), \\
& \sum_{k=1}^{K} \operatorname{tr}\left(\boldsymbol{D}_{W, s, k}\right) \leq P_{s}, \\
& \operatorname{tr}\left(\boldsymbol{D}_{W, r, k}\right) \leq P_{r, k}\left(\alpha, \boldsymbol{S}_{e}\right), \\
& \operatorname{tr}\left(\boldsymbol{S}_{e}\right) \leq P_{s}, \\
& \boldsymbol{D}_{W, s, k} \geq 0, \boldsymbol{D}_{W, r, k} \geq 0, \boldsymbol{S}_{e} \geq 0 .
\end{aligned}
$$

This problem is convex and can be solved using the CVX software in Matlab. Once $\boldsymbol{D}_{W, s, k}$ and $\boldsymbol{D}_{W, r, k}$ solutions to (P3) are obtained, the precoding matrices $\boldsymbol{W}_{s, k}$ and $\boldsymbol{W}_{r, k}$ (or equivalently the covariance matrices $\boldsymbol{S}$ and $\left.\boldsymbol{R}_{r, k}\right)$, for $k=1, \ldots, K$, can be obtained using (26) and (37), respectively.

\section{Remarks and Special Cases}

In this part, we present some special cases (no CCI at the $D_{k} \mathrm{~s}$ ) and standard solutions (TDMA, MMSE) that serve as a benchmark or as a comparison reference for our proposed solution based on the BD method.

1) No Interference Between the Destinations: Here, we assume that the source use the $\mathrm{BD}$ technique and we assume that the destinations are far away from each other in a way to have no interference between the destinations, for the sake of simplicity, each relay $R_{k}$ will be communicating in parallel with the destination $D_{k}$ without cross-link interference. In other words, we have $\boldsymbol{M}_{-k}=\sigma_{d, k}^{2} \boldsymbol{I}_{N_{d, k}}$. Consequently, the objective function in (P1) becomes concave and the optimal $S$ and $\boldsymbol{R}_{r, k}$ become solutions to

$$
\begin{aligned}
\text { (P5) : } \max _{\boldsymbol{S}_{e}, \boldsymbol{D}_{W, s, k}, \boldsymbol{R}_{r, k}} & \sum_{k=1}^{K} \frac{1-\alpha}{2} t_{k}, \\
\text { s.t. } & t_{k} \leq \bar{R}_{S-R_{k}}\left(\alpha, \boldsymbol{D}_{W, s, k}\right), \\
& t_{k} \leq \log _{2}\left|\boldsymbol{I}+\frac{1}{\sigma_{d, k}^{2}} \boldsymbol{G}_{k, k} \boldsymbol{R}_{r, k} \boldsymbol{G}_{k, k}^{H}\right|, \\
& \sum_{k=1}^{K} \operatorname{tr}\left(\boldsymbol{D}_{W, s, k}\right) \leq P_{s}, \\
& \operatorname{tr}\left(\boldsymbol{R}_{r, k}\right) \leq P_{r, k}\left(\alpha, \boldsymbol{S}_{e}\right), \\
& \operatorname{tr}\left(\boldsymbol{S}_{e}\right) \leq P_{s}, \\
& \boldsymbol{D}_{W, s, k} \geq 0, \boldsymbol{R}_{r, k} \geq 0, \boldsymbol{S}_{e} \geq 0 .
\end{aligned}
$$

This equivalent problem is convex and can be solved using the convex optimization tools available in Matlab such as the CVX software [22]. The sum-rate solution of this problem can be seen as an upper bound for the sum-rate solution of the original problem.

2) TDMA Based Solution: Another method to solve (P) is to consider the time division multiple access (TDMA) transmission method at the source and the destinations. In other words, the information transmissions from the source to the relays and the information transmissions from the relays to the destinations are all separated over the time. Let $\frac{1-\alpha}{2} \tau_{k}$ be the duration of the time slot allocated to transmit from the source to the $k^{\prime}$ th relay $R_{k}$ and from the $k^{\prime}$ th relay $R_{k}$ to the $k^{\prime}$ th destination $D_{k}$, where $0 \leq \tau_{k} \leq 1$. In this case, no cross interference will occur at the relays and at the destinations. Thus, there is no need to use neither the BD method at the source nor the interference mitigations methods at the destinations. However, the time allocated for each transmission is reduced. Note that $\tau_{k}$ is optimized in a similar way as $\alpha$.

3) MMSE Based Solution: The sum rate maximization problem of the MIMO interfering broadcast channel was shown to be connected to the minimization of mean-square error (MSE) problem [12]-[15]. Even though our objective function is the sum of the minimum between two rates and is not among the general utility functions described in [13], we propose to investigate the iterative MMSE approach to show the relevance of our proposed method based on the BD at the source and the relays.

First, we perform the iterative MMSE between the source and the relays. The MMSE precoder at the source and the MMSE decoder at the $k^{\prime}$ th relay can be written as [12], [13]

$$
\boldsymbol{W}_{s, k}=\left(\mu_{0} \boldsymbol{I}_{N_{s}}+\boldsymbol{H}_{k}^{H}\left(\sum_{l=1}^{K} \boldsymbol{U}_{r, l} \boldsymbol{U}_{r, l}^{H}\right) \boldsymbol{H}_{k}\right)^{-1} \boldsymbol{H}_{k}^{H} \boldsymbol{U}_{r, k},
$$

and

$$
\boldsymbol{U}_{r, k}=\left(\sigma_{r}^{2} \boldsymbol{I}_{N_{r, k}}+\boldsymbol{H}_{k}\left(\sum_{l=1}^{K} \boldsymbol{W}_{s, l} \boldsymbol{W}_{s, l}^{H}\right) \boldsymbol{H}_{k}\right)^{-1} \boldsymbol{H}_{k} \boldsymbol{W}_{s, k},
$$

where $\mu_{0}$ is the Lagrange multiplier satisfying $\sum_{l=1}^{K} \operatorname{tr}\left(\boldsymbol{W}_{s, l} \boldsymbol{W}_{s, l}^{H}\right)=P_{s}$. The corresponding MSE matrix at the $k^{\prime}$ th relay is given by

$$
E_{S-R_{k}}^{M M S E}\left(\boldsymbol{W}_{s}\right)=\boldsymbol{I}-\boldsymbol{W}_{s, k}^{H} \boldsymbol{H}_{k}^{H} \boldsymbol{J}_{s, k}^{-1} \boldsymbol{H}_{k} \boldsymbol{W}_{s, k},
$$

where

$$
\boldsymbol{J}_{s, k}=\sigma_{r, k}^{2} \boldsymbol{I}_{N_{r, k}}+\boldsymbol{H}_{k}\left(\sum_{l=1}^{K} \boldsymbol{W}_{s, l} \boldsymbol{W}_{s, l}^{H}\right) \boldsymbol{H}_{k} .
$$

Thus, using the MMSE precoder and decoder in (43) and (44), the achievable rate between the source and the $k$ 'th relay can be rewritten as

$$
\tilde{R}_{S-R_{k}}\left(\alpha, \boldsymbol{W}_{s}\right)=-\frac{1-\alpha}{2} \log _{2}\left|E_{S-R_{k}}^{M M S E}\left(\boldsymbol{W}_{s}\right)\right| .
$$

Then, we perform the iterative MMSE between the $K$ relays and the $K$ destinations. For $k=1, \ldots, K$, the MMSE precoder at the $k^{\prime}$ th relay and the MMSE decoder at the $k^{\prime}$ th destination are given by [12], [13]

$$
\boldsymbol{W}_{r, k}=\left(\mu_{k} \boldsymbol{I}_{N_{r, k}}+\sum_{l=1}^{K} \boldsymbol{G}_{l, k}^{H} \boldsymbol{U}_{d, l} \boldsymbol{U}_{d, l}^{H} \boldsymbol{G}_{l, k}\right)^{-1} \boldsymbol{G}_{k, k}^{H} \boldsymbol{U}_{d, k},
$$

and

$$
\boldsymbol{U}_{d, k}=\left(\sigma_{d, k}^{2} \boldsymbol{I}_{N_{d, k}}+\sum_{l=1}^{K} \boldsymbol{G}_{k, l} \boldsymbol{W}_{r, l} \boldsymbol{W}_{r, l}^{H} \boldsymbol{G}_{k, l}^{H}\right)^{-1} \boldsymbol{G}_{k, k} \boldsymbol{W}_{r, k},
$$

where $\mu_{k}$ is the Lagrange multipliers satisfying $\operatorname{tr}\left(\boldsymbol{W}_{r, k} \boldsymbol{W}_{r, k}^{H}\right)=$ $P_{r, k}\left(\alpha, S_{e}\right)$. The values of $P_{r, k}\left(\alpha, S_{e}\right)$ are the ones obtained from the maximization of the sum of harvested energies at the $K$ relays. The corresponding MSE matrix at the $k^{\prime}$ th destination is given by

$$
E_{R_{k}-D_{k}}^{M M S E}\left(\boldsymbol{W}_{r}\right)=\boldsymbol{I}-\boldsymbol{W}_{r, k}^{H} \boldsymbol{G}_{k, k}^{H} \boldsymbol{J}_{d, k}^{-1} \boldsymbol{G}_{k, k} \boldsymbol{W}_{r, k},
$$




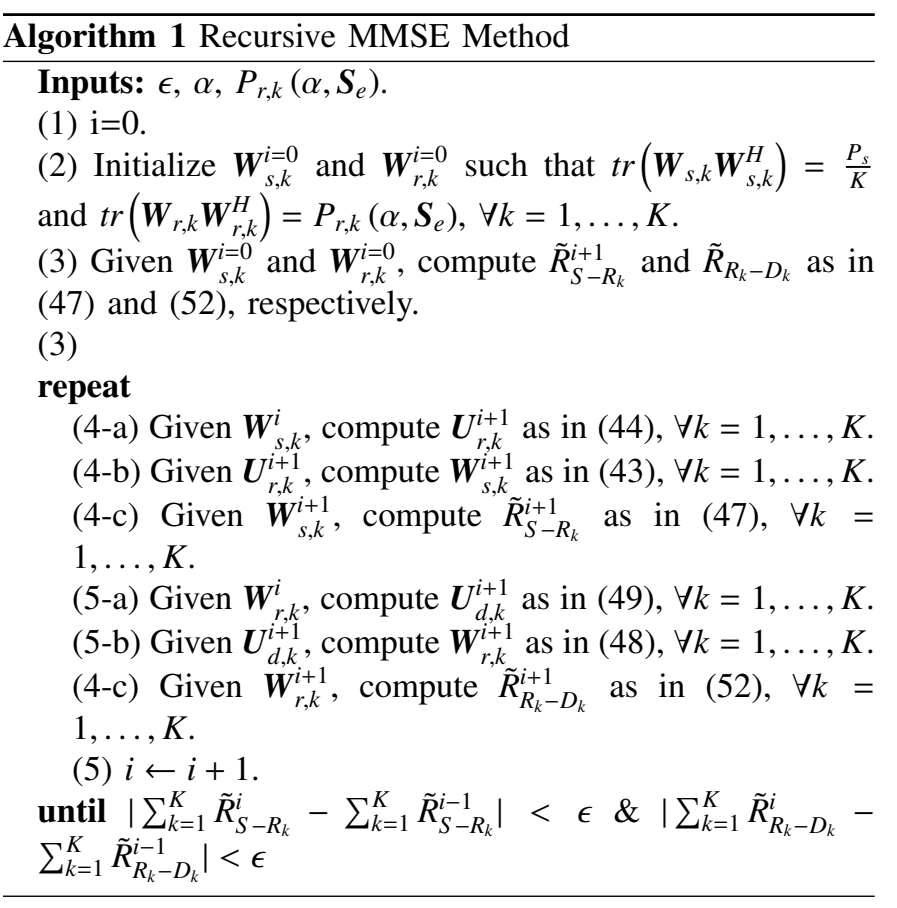

where

$$
\boldsymbol{J}_{d, k}=\sigma_{d, k}^{2} \boldsymbol{I}_{N_{d, k}}+\sum_{l=1}^{K} \boldsymbol{G}_{k, l} \boldsymbol{W}_{r, l} \boldsymbol{W}_{r, l}^{H} \boldsymbol{G}_{k, l}^{H} .
$$

Thus, using the MMSE precoder and decoder in (48) and (49), the achievable rate between the $k^{\prime}$ th relay and the $k^{\prime}$ th destination can be rewritten as

$$
\tilde{R}_{R_{k}-D_{k}}\left(\alpha, \boldsymbol{W}_{r}\right)=-\frac{1-\alpha}{2} \log _{2}\left|E_{R_{k}-D_{k}}^{M M S E}\left(\boldsymbol{W}_{r}\right)\right| .
$$

The iterative MMSE for the multiuser MIMO DF relay broadcasting channel is resumed in Algorithm 1. The optimal $\alpha$ is selected in a similar way as in the BD based solution, by a one-dimensional search method. For each $\alpha$, we have the harvested energy at each relay is initialized to the one obtained from the BD solution.

\section{Numerical Results}

In this section, we present some numerical results related to the sum rate maximization problem addressed in this paper. We consider the case where the channels between the source and the relays and the channels between the relays and the destinations are fading channels with path loss. The path loss exponent is chosen equal to $m=2.7$ which corresponds to an urban cellular network environment [4]. The multiuser MIMO relay broadcasting communication system is composed of one source, two relays, and two destinations. The number of transmit antennas at the source are equal to $N_{s}=6$. The number of transmit antennas at the relays are equal to $N_{r, 1}=N_{r, 2}=3$. The number of transmit antennas at the destinations are equal to $N_{d, 1}=N_{d, 2}=2$. We denote by $d_{s r}, d_{r d}$ and $d_{s d}$ the distance between the source and relays, the distance between the relays and destinations and the distance between the source and destinations, respectively. In the simulations, we assume that all the relays are equidistant from the source, as well as all the destinations. As shown

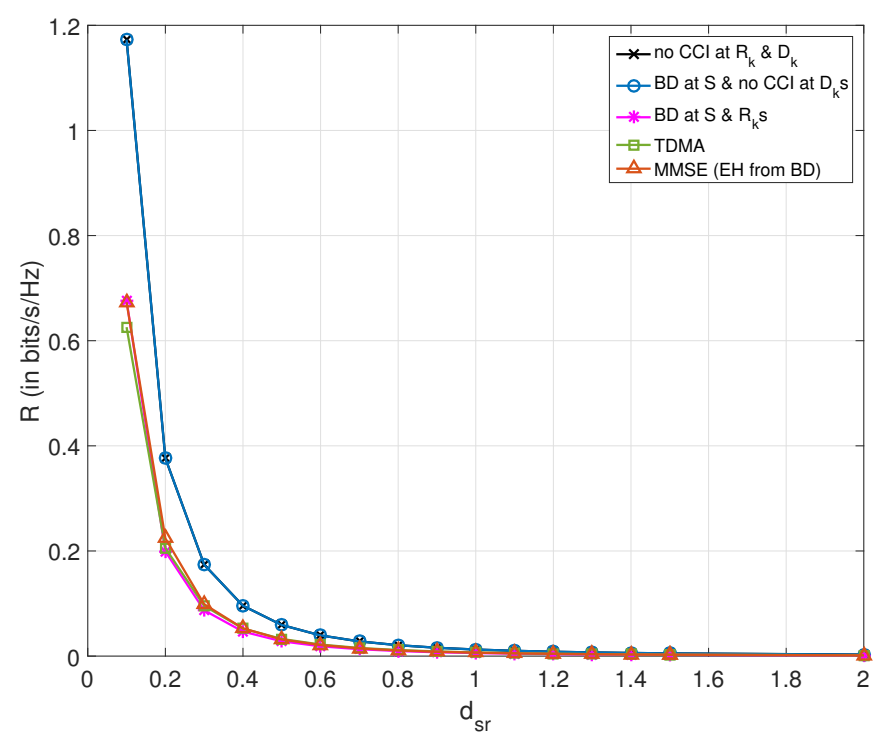

Figure 2. The sum of the source-destinations rates versus the source-relays distance $d_{s r}$.

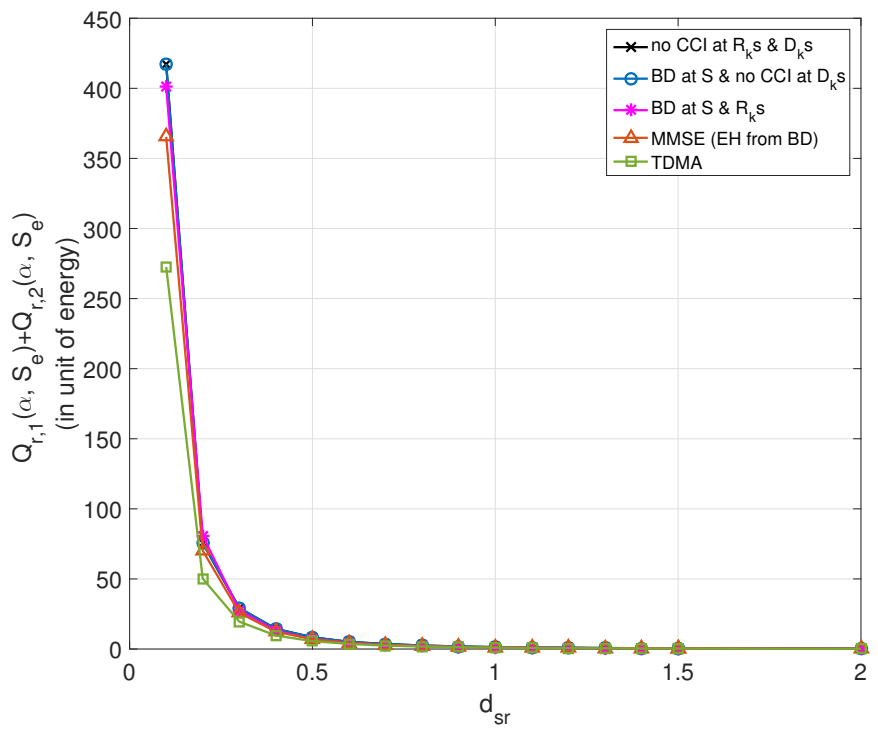

Figure 3. The sum of the harvested energy at the relays versus the sourcerelays distance $d_{s r}$.

in [23], [24], the wireless energy transfer is limited by the distance between the transmitter and the receiver. Thus, in our simulations, we are limited to cases of short distances, in order of meters. The cases of long distances will be the object of the extension of this work. We assume also that $\sigma_{r, k}^{2}=\sigma_{d, k}^{2}=1$, $\forall k=1, \ldots, K$. The conversion efficiency at the $\mathrm{EH}$ receivers at all relays is chosen equal to $\zeta=1$.

In Figs. 2 and 3and 4, we have plotted the sum of the endto-end rates, the sum of the harvested energy at the relays, and the corresponding time ratio allocated for the EH mode, versus the distance between the source and the relays $d_{s r}$. The transmit power at the source is equal to $-10 \mathrm{dBW}$. The 


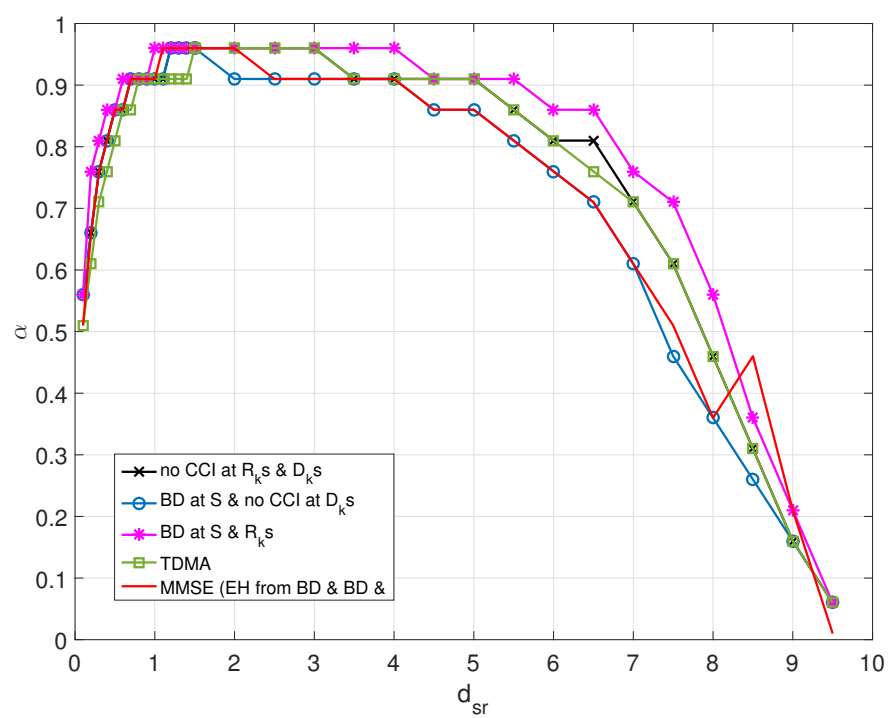

Figure 4. The optimal time ratio $\alpha$.

proposed solution in the paper are depicted where we perform the $\mathrm{BD}$ procedure to cancel the interference at the relays, and the interference cancellation methods at the destinations based on the $\mathrm{BD}$ procedure. The proposed solution is compared to ideal cases and other standard solutions derived in III-D that serves as a benchmark for our simplified solution:

(a) no $\mathrm{CCI}$ occurs at the relays and the destinations,

(b) the $\mathrm{BD}$ procedure is performed at the source and no $\mathrm{CCI}$ occurs at the destinations.

(c) The TDMA based solution is performed where $\tau_{k}$ 's are all optimized.

(d) The MMSE based solution where the harvested energies at the relays are those obtained by our proposed solution. The covariance matrices $\boldsymbol{S}_{e}$ cannot be jointly obtained as the simplified problem using the MMSE solution in not convex.

We can see that our simplified solution provides sum-rates closer to the TDMA and MMSE solutions. Note that the optimal value of $\tau_{1}$ is greater than 0.7 and increases to 1 as we increase $d_{s r}$. We can see that the end-to-end sum-rate and the sum of harvested energies at the $K$ relays decrease as we increase the distance between the source and the relays which is in accordance with the fact that wireless energy transfer is more effective over short distances. The sum of harvested energies at the $K$ relays corresponding to our proposed solution is greater than those of the other solutions.

In Figs. 5 and 6 and 7, we have plotted the sum of the endto-end rates and the sum of the harvested energy at the relays, and the corresponding time ratio allocated for the $\mathrm{EH}$ mode versus the transmit power at the source in $\mathrm{dBW}$. The relays are at a distance equal to $d_{s r}=0.15 d_{s d}$ where $d_{s d}=5 \mathrm{~m}$. The end-to-end rates and the sum of the harvested energies at the $K$ relays of all the considered solutions increase as we increase the transmit power at the source $P_{s}$, while the time ratio $\alpha$ decreases. We can see that all the interference cancellation

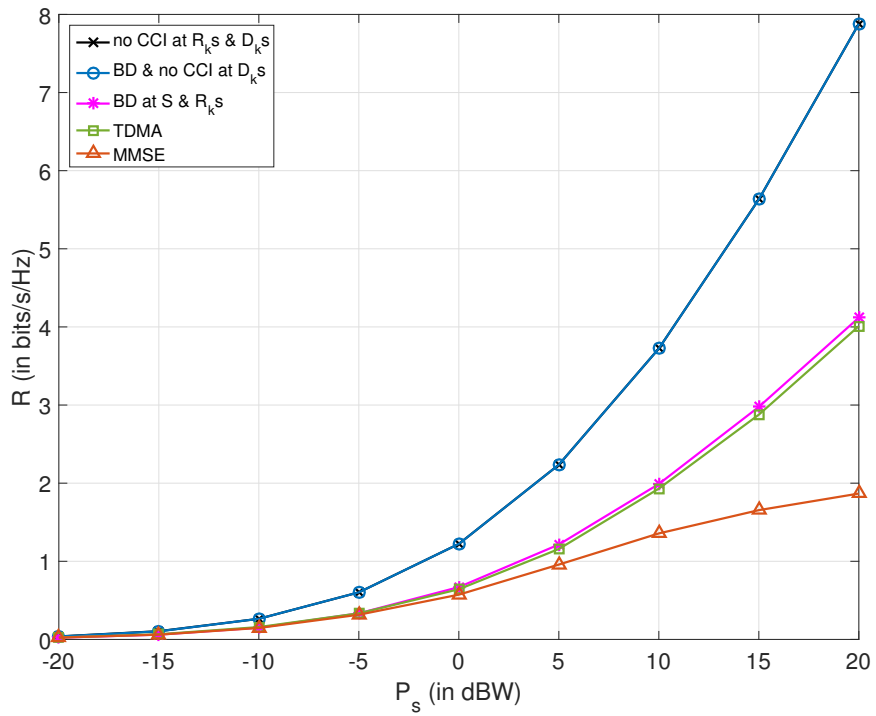

Figure 5. The sum of the source-destinations rates versus the transmit power at the source $P_{s}$.

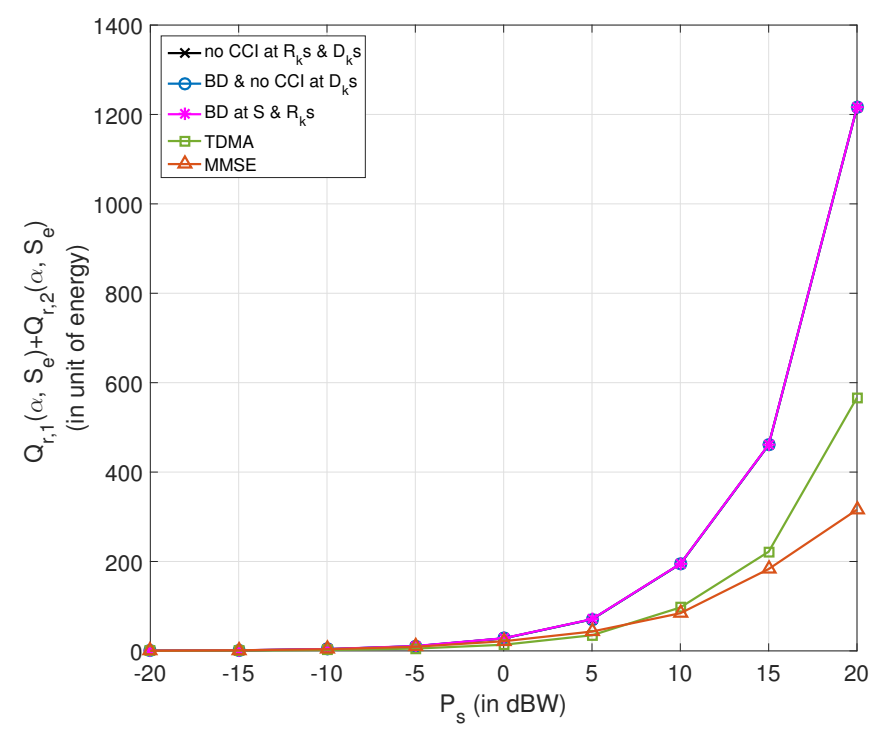

Figure 6. The sum of the harvested energy at the relays versus the transmit power at the source $P_{s}$.

methods at the destinations have close performance for $P_{s}<0$ $\mathrm{dBW}$. Otherwise, the TDMA transmission and the BD method outperform the MMSE based solution. On the other hand, the no CCI case and the BD method require more harvested energy at the relays than the TDMA and the MMSE solutions.

\section{Conclusion}

In this paper, we have considered the sum-rate enhancement problem in multiuser MIMO DF relay broadcasting channel with EH relays. We have solved the simplified problem where we perform the $\mathrm{BD}$ method at the source to eliminate the interference at the relays, and we use interference mitigation methods to cancel the interference between the relaydestination channels. We have shown the relevance of our 


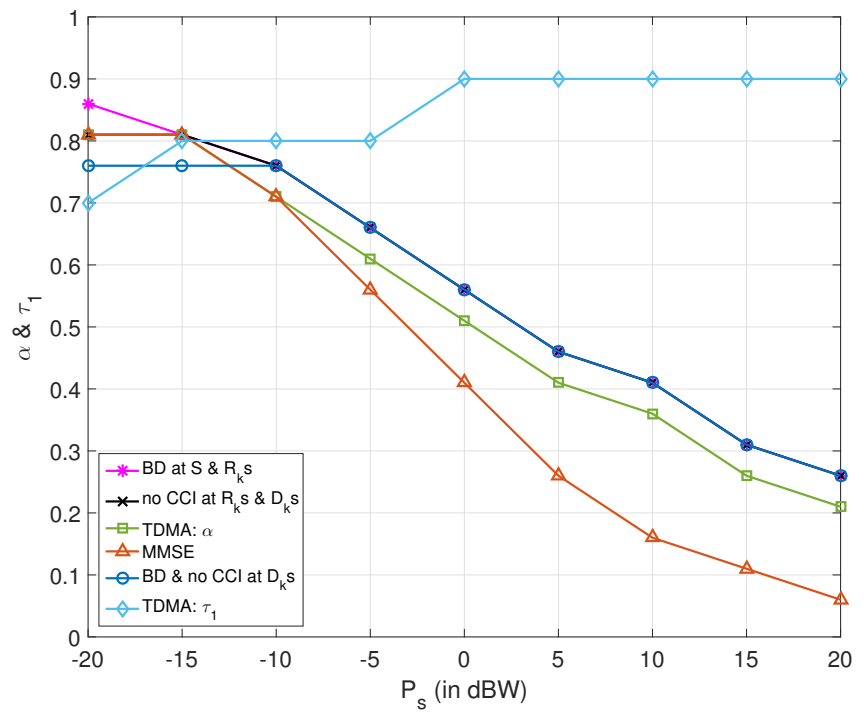

Figure 7. The optimal time ratio $\alpha$ and $\tau_{1}$ (for TDMA).

proposed solution through some selected numerical results in comparison with some special cases and standard solutions like the MMSE and TDMA.

\section{REFERENCES}

[1] L. Varshney, "Transporting information and energy simultaneously," in IEEE International Symposium on Information Theory (ISIT'2008), Jul. 2008, pp. 1612-1616.

[2] P. Grover and A. Sahai, "Shannon meets Tesla: Wireless information and power transfer," in IEEE International Symposium on Information Theory Proceedings (ISIT'2010), Jun. 2010, pp. 2363-2367.

[3] R. Zhang and C. K. Ho, "MIMO broadcasting for simultaneous wireless information and power transfer," IEEE Transactions on Wireless Communications, vol. 12, no. 5, pp. 1989-2001, May 2013.

[4] A. Nasir, X. Zhou, S. Durrani, and R. Kennedy, "Relaying protocols for wireless energy harvesting and information processing," IEEE Transactions on Wireless Communications, vol. 12, no. 7, pp. 3622-3636, Jul. 2013.

[5] A. A. Nasir, X. Zhou, S. Durrani, and R. A. Kennedy, "Wireless energy harvesting and information relaying: Adaptive time-switching protocols and throughput analysis," CoRR, vol. abs/1310.7648, 2013.

[6] B. Chalise, W.-K. Ma, Y. Zhang, H. Suraweera, and M. Amin, "Optimum performance boundaries of OSTBC based AF-MIMO relay system with energy harvesting receiver," IEEE Transactions on Signal Processing, vol. 61, no. 17, pp. 4199-4213, Sept. 2013.

[7] A. Nasir, X. Zhou, S. Durrani, and R. Kennedy, "Throughput and ergodic capacity of wireless energy harvesting based DF relaying network," in IEEE International Conference on Communications (ICC'2014), Jun. 2014, pp. 4066-4071.
[8] G. Caire and S. Shamai, "On the achievable throughput of a multiantenna gaussian broadcast channel," IEEE Transactions on Information Theory, vol. 49, no. 7, pp. 1691-1706, Jul. 2003.

[9] W. Yu and J. Cioffi, "Sum capacity of gaussian vector broadcast channels," IEEE Transactions on Information Theory, vol. 50, no. 9 , pp. 1875-1892, Sept. 2004

[10] H. Weingarten, Y. Steinberg, and S. Shamai, "The capacity region of the Gaussian multiple-input multiple-output broadcast channel," IEEE Transactions on Information Theory, vol. 52, no. 9, pp. 3936-3964, Sept. 2006.

[11] Q. Spencer, A. Swindlehurst, and M. Haardt, "Zero-forcing methods for downlink spatial multiplexing in multiuser MIMO channels," IEEE Transactions on Signal Processing, vol. 52, no. 2, pp. 461-471, Feb. 2004.

[12] S. W. Peters and R. W. Heath, "Cooperative algorithms for MIMO interference channels," IEEE Transactions on Vehicular Technology, vol. 60, no. 1, pp. 206-218, Jan. 2011.

[13] Q. Shi, M. Razaviyayn, Z. Q. Luo, and C. He, "An iteratively weighted MMSE approach to distributed sum-utility maximization for a MIMO interfering broadcast channel," IEEE Transactions on Signal Processing, vol. 59, no. 9, pp. 4331-4340, Sept. 2011.

[14] F. Negro, S. P. Shenoy, I. Ghauri, and D. T. M. Slock, "On the MIMO interference channel," in Information Theory and Applications Workshop (ITA), 2010, Jan. 2010, pp. 1-9.

[15] S. S. Christensen, R. Agarwal, E. D. Carvalho, and J. M. Cioffi, "Weighted sum-rate maximization using weighted MMSE for MIMOBC beamforming design," IEEE Transactions on Wireless Communications, vol. 7, no. 12, pp. 4792-4799, Dec. 2008.

[16] L. Liu, R. Zhang, and K.-C. Chua, "Multi-antenna wireless powered communication with energy beamforming," IEEE Transactions on Communications, vol. 62, no. 12, pp. 4349-4361, Dec. 2014.

[17] G. Yang, C. K. Ho, R. Zhang, and Y. L. Guan, "Throughput optimization for massive MIMO systems powered by wireless energy transfer," CoRR, vol. abs/1403.3991, 2014. [Online]. Available: http://arxiv.org/abs/1403.3991

[18] J. Park and B. Clerckx, "Joint wireless information and energy transfer in a K-user MIMO interference channel," IEEE Transactions on Wireless Communications, vol. 13, no. 10, pp. 5781-5796, Oct. 2014.

[19] X. Zhou, R. Zhang, and C. K. Ho, "Wireless information and power transfer: Architecture design and rate-energy tradeoff," IEEE Transactions on Communications, vol. 61, no. 11, pp. 4754-4767, Nov. 2013.

[20] G. Scutari, D. Palomar, and S. Barbarossa, "The MIMO iterative waterfilling algorithm," IEEE Transactions on Signal Processing, vol. 57, no. 5, pp. 1917-1935, May 2009.

[21] S. Boyd and L. Vandenberghe, Convex Optimization. New York, NY, USA: Cambridge University Press, 2004.

[22] M. Grant and S. Boyd, "CVX: Matlab software for disciplined convex programming." [Online]. Available: http://stanford.edu/ boyd/cvx

[23] D. W. K. Ng and R. Schober, "Secure and green SWIPT in distributed antenna networks with limited backhaul capacity," IEEE Transactions on Wireless Communications, vol. 14, no. 9, pp. 5082-5097, 2015.

[24] C. Zhai and J. Liu, "Cooperative wireless energy harvesting and information transfer in stochastic networks," EURASIP Journal on Wireless Communications and Networking, vol. 2015, no. 1, p. 44, 2015.

[Online]. Available: http://jwcn.eurasipjournals.com/content/2015/1/44 